\title{
Tumor mesenquimatoso fosfatúrico de fosa nasal con compromiso intracraneano: Reporte de un caso y revisión de la literatura
}

\section{Nasal fossa phosphaturic mesenchymal tumor with intracraneal compromise, case report and review of the literature}

\author{
Tomás Andrade $D^{1}$, Fernando Slater $\mathbf{R}^{2}$, Claudia González G², Rodrigo Cabezón A².
}

\begin{abstract}
RESUMEN
El tumor mesenquimatoso fosfatúrico (TMF) es una enfermedad extremadamente rara. Según evidencia reciente es causado por la sobreexpresión del factor de crecimiento fibroblástico 23 (FGF23), el cual genera hipofosfemia y osteomalacia. A continuación presentamos el caso de un paciente de 42 años con un tumor mesenquimatoso fosfatúrico de fosa nasal izquierda con extenso compromiso intracraneano. Cabe destacar que hasta la fecha hay 142 casos reportados de TMF en la literatura de los cuales solo 11 se ubican en fosa nasal y cavidades sinusales, y sólo dos de ellos ubicados en fosa nasal'.

El paciente tuvo una exitosa resolución quirúrgica con la consecuente normalización de parámetros analíticos (incluido el FGF23), mejoría sintomática y ausenia de recidiva hasta la fecha.
\end{abstract}

Palabras clave: Tumor mesenquimatoso fosfatúrico; osteomalacia oncogénica; factor de crecimiento fibroblástico 23; tumor inductor de osteomalacia; hipofosfemia.

\begin{abstract}
The phosphaturic mesenchymal tumor (PMT) is an extremely rare disease. According to recent evidence is caused by overexpression of fibroblast growth factor 23 (FGF23) which generates hypophosphatemia and osteomalacia. We report the case of a 42 year old patient with a left nasal fossa phosphaturic mesenchymal tumor with intracranial involvement. Should be noted that to date there are 142 reported cases of PMT in the literature of which only 11 are located in nasal fossa and sinus cavities, two of them located in nasal fossa'.

The patient had a successful surgical resolution with consequent normalization of analytical parameters (including FGF23), absence of symptoms and no recurrence to date.
\end{abstract}

Key words: Phosphaturic mesenchymal tumor; oncogenic osteomalacia; fibroblast growth factor 23; tumor-induced osteomalacia; hypophosphatemia.

Médico-Cirujano. Facultad de Medicina, Pontificia Universidad Católica de Chile.

2 Médico. Departamento Otorrinolaringología, Pontificia Universidad Católica de Chile. 


\section{INTRODUCCIÓN}

Los tumores mesenquimatosos fosfatúricos (TMF) corresponden a uno de varios tipos denominados tumores inductores de osteomalacia (TIO), los cuales pueden causar osteomalacia oncogénica ${ }^{2-7}$. Esta corresponde a una rara enfermedad paraneoplásica adquirida caracterizada por la pérdida renal de fósforo con la consecuente hipofosfemia. Evidencia reciente muestra que la sobreexpresión tumoral de un factor de crecimiento fibroblástico (FGF23), el cual regula los niveles de vitamina $D$ y fósforo plásmatico sería el responsable de la hipofosfemia, niveles bajos de 1,23dihidroxivitamina-D y la consecuente osteomalacia ${ }^{8}$.

EI TIO fue descrito por primera vez en 1947 por McCance 9 , y se han reportado en la literatura aproximadamente 300 casos hasta la fecha ${ }^{3}$, de estos 142 corresponden a TMF ${ }^{1}$ y sólo 11 de ellos se ubican en cavidades nasales 0 fosa nasal $\left.\right|^{1,13}$ como es el caso de nuestro paciente.

A continuación presentamos el caso clínico de un paciente portador de un TMF que fue tratado en nuestro centro, y revisión de la literatura del tema.

\section{CASO CLÍNICO}

Se reporta el caso de un paciente de género masculino de 42 años, casado, residente en la Región de Antofagasta, con antecedentes de dislipidemia mixta y tabaquismo crónico. Desde el año 2006 consulta por cuadro de meses de evolución de dolor lumbar, fatigabilidad, baja de peso de $20 \mathrm{~kg}$, fracturas y pseudofracturas múltiples (costales, tobillo, pelvis, entre otras). Se estudia en ese momento por endocrinología en su región, llegando al diagnóstico de osteomalacia hipofosfémica fosfatúrica, descartándose insuficiencia renal, por lo que recibe tratamiento con fosfato monosódico, vitamina $D$ y antiinflamatorios no esteroidales (AINES), sumado a los fármacos habituales (simbastatina y ciprofibrato) para su comorbilidad. Sin embargo, el tratamiento farmacológico no logra evitar la progresión de la enfermedad y su sintomatología.

En el 2011 el paciente presenta una lesión de crecimiento progresivo en codo izquierdo, lo que hace sospechar a su equipo tratante la posibilidad de una causa oncogénica que explicara la osteomalacia, por lo que se realiza biopsia excisional de esta lesión (agosto 2011), la cual fue compatible con un hemangiolipoma.
La osteomalacia persistió sin cambios. Con este antecedente, y dada la historia concomitante de obstrucción nasal izquierda persistente de 5 años de evolución, se inicia estudio por otorrinolaringología. Se realiza estudio de masa en fosa nasal izquierda en el Hospital Regional de Antofagasta, donde se realiza estudio imagenológico (descrito a continuación), y 2 biopsias endoscópicas, la primera con resultado no concluyente, y la segunda con histología compatible con histiocitoma fibroso benigno. Dados estos hallazgos, el paciente es derivado a nuestro centro, para resolución quirúrgica, y manejo metabólico.

En su primera evaluación se observa un paciente pálido, enflaquecido, con marcha claudicante. La rinoscopía muestra una masa polipoidea en fosa nasal comprometida, sin rinorrea, epistaxis, rinorraquia ni otros hallazgos de importancia al examen otorrinolaringológico. Teniendo en conocimiento todos los antecedentes anteriormente expuestos, se solicitan exámenes generales, se actualizan exámenes imagenológicos y se presenta el caso en comité interdisciplinario entre otorrinolaringología, neurocirugía y endocrinología.

Los exámenes de laboratorio prequirúrgicos se presentan en la Tabla 1, destacando la paratohormona (PTH) y VHS elevadas, y la hipofosfemia refractaria pese al uso permanente de suplementos con fosfato.

La TC de cavidades perinasales muestra un tumor con densidad de partes blandas centrado en FNI, con desplazamiento de tabique nasal a derecha, ocupación de seno frontal, esfenoidal y maxilar ipsilateral, focos de densidad grasa en su espesor y extensión hacia superior con destrucción

\section{Tabla 1. Exámenes de laboratorio prequirúrgicos}

\begin{tabular}{|ll|}
\hline Exámenes de laboratorio & Resultados \\
\hline FGF 23 & $75,98 \mathrm{pg} / \mathrm{mL}(8,2-54,3)$ \\
PTH (paratohormona) & $93 \mathrm{pg} / \mathrm{ml}(15-75)$ \\
VHS & $92 \mathrm{~mm} / \mathrm{hr}$ \\
Hematocrito & $37 \% \mathrm{Hb} 12,2 \mathrm{~g} / \mathrm{dl}$ \\
Creatinina & $0,83 \mathrm{mg} / \mathrm{dl}$ \\
Fosfatasas alcalinas & $217 \mathrm{U} / \mathrm{L}(45-115)$ \\
Calcio & $9,2 \mathrm{mg} / \mathrm{dl}(8,5-10,5)$ \\
Fósforo & $1,7 \mathrm{mg} / \mathrm{dl}(2,6-4,5)$ \\
Fósforo urinario & $0,6 \mathrm{~g} / 24 \mathrm{hrs}(0,3-1)$ \\
BUN & $30 \mathrm{mg} / \mathrm{dl}$ \\
Proteínas totales & $7,2 \mathrm{mg} / \mathrm{dl}$ \\
Calcio iónico & $4,6 \mathrm{mg} / \mathrm{dl}$ \\
\hline
\end{tabular}




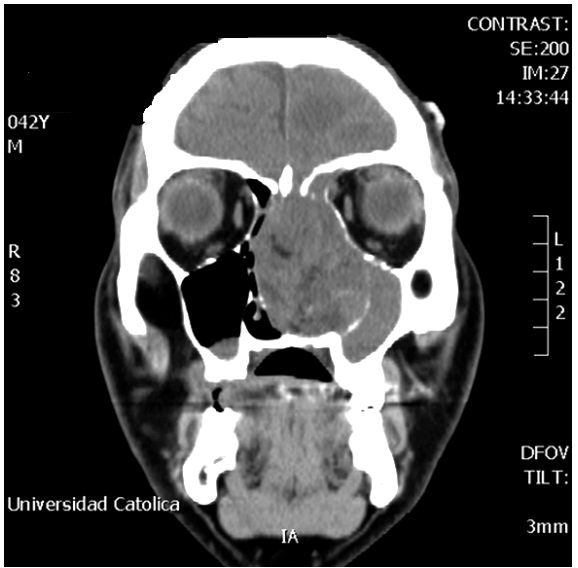

Figura 1.

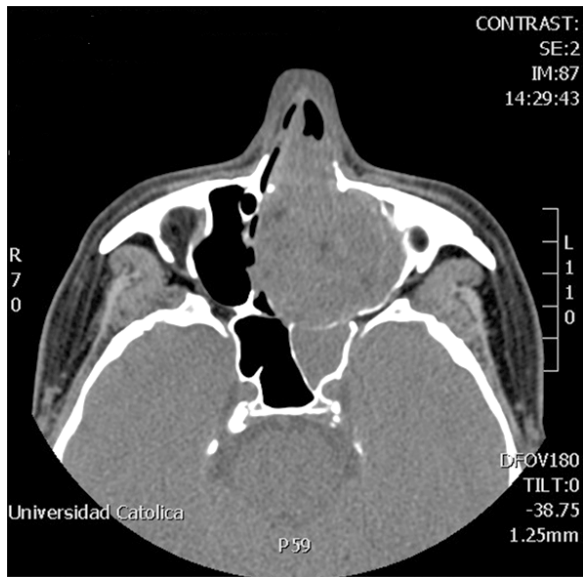

Figura 2.

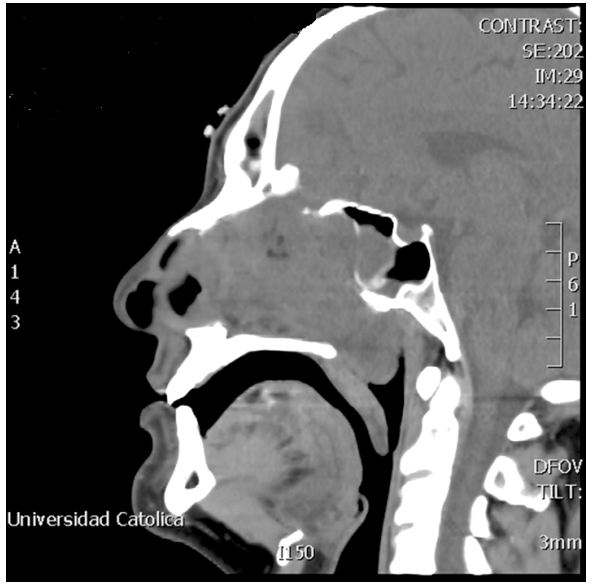

Figura 3.

de la lámina cribiforme, y extensión posterior a nasofaringe, con aspecto radiológico compatible con un estesioneuroblastoma (Figuras 1-3). Se complementa estudio con RM de cerebro que confirma infiltración de lámina cribiforme y extensa invasión tumoral intracraneana, también con impresión radiológica de un tumor nasofrontal compatible con estesioneuroblastoma (Figuras 4-6).

Dados los antecedentes del paciente, imágenes e histología, se plantea la hipótesis diagnóstica de osteomalacia oncogénica por tumor fosfatúrico nasofrontal. Se programa cirugía en junio del presente año, realizando un abordaje combinado, con una fase neuroquirúrgica con abordaje bicoronal y craneotomía anterior, resección de la porción frontal del tumor, desfuncionalización del seno frontal y cierre del defecto

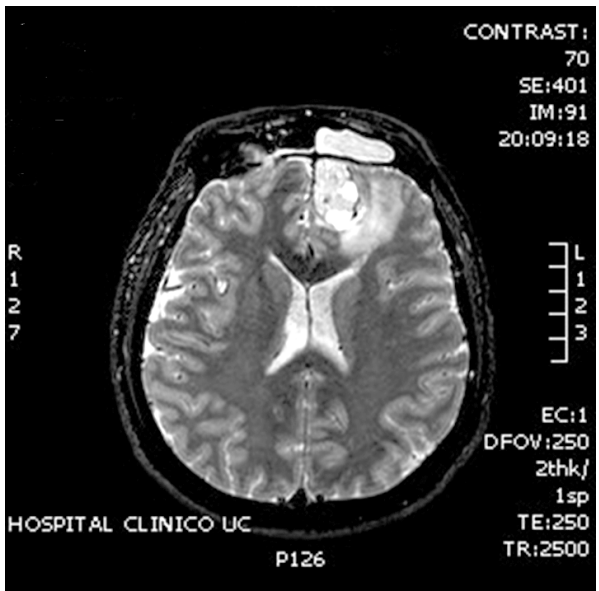

Figura 4

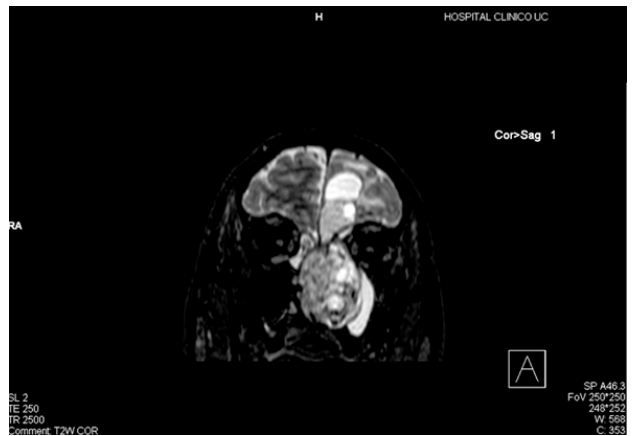

Figura 5.

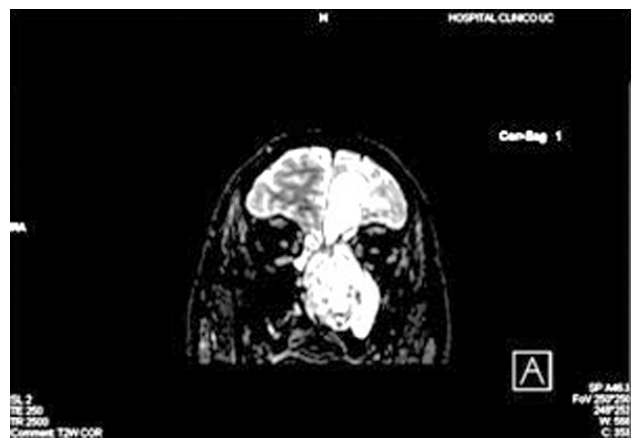

Figura 6. 
de fosa anterior utlilizando placa de titanio, y una fase endonasal, donde se completa la resección del tumor, y se realiza una antrostomía maxilar y esfenoidotomía amplia izquierda. Al revisar techo etmoidal se visualiza fístula de líquido céfalo raquídeo posterior a placa de titanio antes mencionada, por lo que se cubre el defecto y la placa con un colgajo nasoseptal de Haddad con pedículo septal posterior izquierdo. Se finaliza de esta forma la cirugía sin incidentes.

El paciente presenta un posoperatorio favorable, normalizando su fósforo plasmático al tercer día, y el resultado de la biopsia corresponde a un tumor mesenquimático osificante benigno, confirmando de esta forma el diagnóstico de tumor mesenquimatoso fosfatúrico (Figura 7). El paciente es dado de alta a los 6 días, y ha presentado una notable mejoría de su capacidad funcional. Los resultados de los exámenes de control a los 3 meses del posquirúrgico se presentan en la Tabla 2. Hasta el momento, no ha presentado recidiva.

\section{REVISIÓN DE LA LITERATURA}

El primer caso de un tumor inductor de osteomalacia fue descrito por Robert McCance en 1947, quien reportó un paciente con dolor, debilidad, claudicación de la marcha y niveles bajos de fósforo plasmático. Fue tratado con vitamina $D$ en altas dosis sin obtener respuesta, sin embargo sus síntomas se resolvieron al resecar el tumor femoral que poseía el paciente ${ }^{9}$. Hasta la actualidad existen aproximadamente 300 casos publicados en la literatura ${ }^{3}$, de los cuales 142 corresponden a tumores mesenquimatosos fosfatúricos ${ }^{1}$. La mayoría de estos tumores aparecen en tejidos blandos y huesos, siendo en frecuencia las extremidades la principal localización ${ }^{10,11}$ y en segundo lugar la región de cabeza y cuello $0^{10}$. Hasta la fecha solo existen 11 casos descritos en la literatura de localización en fosa nasal (FN) 0 cavidades sinusales, de los cuales sólo dos están ubicados en $\mathrm{FN}^{1,13}$. El caso que presentamos de TMF nasal correspondería al tercero reportado en la literatura, constituyendo una variante extremadamente rara e infrecuente (Tabla 3).

Como se dijo anteriormente los TMF están enmarcados dentro de los denominados TIO, los cuales son extremadamente infrecuentes y pueden causar un síndrome paraneoplásico denomi-

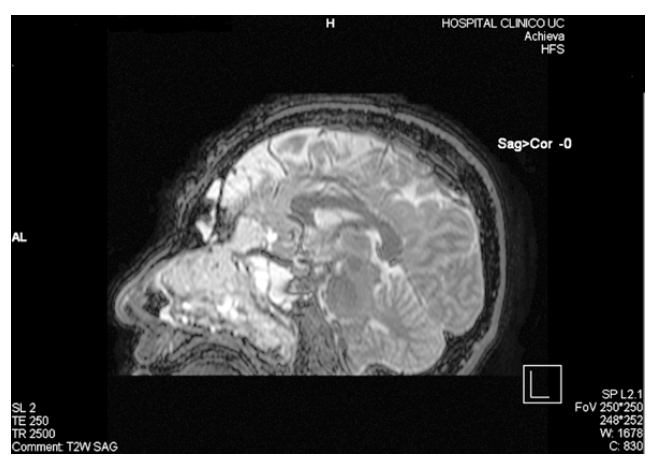

Figura 7. Cortes histológicos que muestran mucosa sinusal infiltrada por tumor no epitelial de patrón fusocelular, con presencia de células multinucleadas de tipo osteoclástico, compatible con un tumor mesenquimático osificante, acorde con el planteamiento clínico de tumor fosfatúrico.

nado osteomalacia oncogénica ${ }^{2-7}$. Esta última corresponde a una rara entidad causada por la sobreexpresión tumoral del FGF 238,11, el cual fue identificado como el último miembro de la familia de factores de crecimiento fibroblástico el año 2011 por Shimada y cols. El FGF23 actúa como un regulador endocrino de la 1,25 dihidroxivitamina-D y del fósforo plasmático ${ }^{8,12,15}$, de tal forma que niveles plasmáticos

\section{Tabla 2. Exámenes de laboratorio a los 3 meses} de la cirugía

\begin{tabular}{|lll|}
\hline Exámenes de laboratorio & \multicolumn{2}{c|}{ Resultados } \\
\hline FGF 23 & $8,4 \mathrm{pg} / \mathrm{mL}$ & $(8,2-54,3)$ \\
Fosfatasas alcalinas & $273 \mathrm{U} / \mathrm{L}$ & $(45-115)$ \\
Calcio & $9,7 \mathrm{mg} / \mathrm{dl}$ & $(8,5-10,5)$ \\
Fósforo & $3,6 \mathrm{mg} / \mathrm{dl}$ & $(2,6-4,5)$ \\
Fósforo urinario & $0,42 \mathrm{~g} / 24 \mathrm{hrs}$ & $(0,3-1)$ \\
\hline
\end{tabular}

Tabla 3. Casos reportados de TMF localizados en fosa nasal y cavidades paranasales ${ }^{3,16-24}$

\begin{tabular}{|c|c|c|}
\hline Referencia & Edad y sexo & Lugar \\
\hline Linsey $1982^{16}$ & $54 \mathrm{~F}$ & Nasofaringe \\
\hline Weidner y Santa Cruz $1987^{17}$ & $35 \mathrm{~F}$ & Seno maxilar \\
\hline Papotti $1988^{18}$ & $38 \mathrm{~F}$ & Cavidad nasal \\
\hline Wilkins $1992^{19}$ & $55 \mathrm{M}$ & Seno maxilar \\
\hline Gonzalez Compta $1998^{20}$ & $69 \mathrm{~F}$ & Seno etmoidal \\
\hline Ohashi 199921 & $43 \mathrm{M}$ & Seno maxilar \\
\hline Clunie $2000^{22}$ & $60 \mathrm{~F}$ & Seno etmoidal \\
\hline Sandhu $2000^{23}$ & $46 \mathrm{M}$ & Seno etmoidal \\
\hline John $2001^{24}$ & $54 \mathrm{~F}$ & Seno maxilar \\
\hline Folpe $2004^{3}$ & $46 \mathrm{M}$ & Seno etmoidal \\
\hline Folpe $2004^{3}$ & $21 \mathrm{M}$ & Seno etmoidal/esfenoidal \\
\hline
\end{tabular}


elevados de este factor endocrino provocan una disminución de la 1aaa- hidroxilasa y aumento de 24hidroxilasa determinando una disminución de la 1,25dihidroxivitamina- D, además inhibe el transporte de fosfato a nivel del túbulo contorneado proximal renal generando la consecuente hipofosfemia e hiperfosfaturia resultando finalmente en la inadecuada mineralización del osteoide en hueso maduro, lo cual corresponde a un desorden metabólico conocido como osteomalacia ${ }^{13,15}$. Es por esto que los marcadores bioquímicos de este desorden son hipofosfemia, niveles inapropiadamente normales 0 bajos de 1,25 dihidroxivitamina-D, y FGF23 elevado en el plasma.

Debido a las alteraciones metabólicas antes mencionadas, la presentación clínica de estos pacientes es dolor de huesos, debilidad muscular, fracturas múltiples, baja de peso significativa y gran compromiso del estado general entre otros ${ }^{10,11,14,18,20,27}$. Dado lo inespecífico y progresivo de los signos y síntomas descritos, y sumado al hecho de que no en todas las instituciones el fósforo plasmático es parte de los análisis de rutina, hay un gran retardo del diagnóstico ${ }^{26}$.

Respecto al tratamiento los TMF y su efecto clínico (osteomalacia oncogénica), están enmarcados dentro de un grupo de neoplasias endocrinas llamativas, ya que al ser resecadas presentan una dramática y satisfactoria resolución ${ }^{14,16,25}$, tal como ocurrió hace seis décadas con el primer caso reportado. Es de vital importancia una resección con márgenes amplios complementándola con una revisión quirúrgica precisa y un seguimiento a largo plazo para detectar posibles recurrencias ${ }^{14,16,20,25,28}$. Pese a que habitualmente son tumores benignos se han descrito casos de transformación maligna y metástasis ${ }^{1,25,28}$ lo cual apoya aún más el seguimiento. Por otro lado, ya que la gran mayoría de los tumores de este tipo son pequeños y difíciles de localizar ${ }^{14,20,25}$, en caso de no encontrar el tumor el tratamiento es médico en base a calcitriol y fosfato.

\section{CONCLUSIONES}

Los TIO son síndromes paraneoplásicos extremadamente infrecuentes causados por la sobreexpresión del FGF23, el cual provoca hipofosfemia, hiperfosfaturia y niveles inapropiados de 1,15 dihidroxivitamina-D, generando síntomas inespecíficos tales como compromiso del estado general, baja de peso, fracturas, claudicación de la marcha, entre otros. Si se localiza el tumor el tratamiento es la exéresis con márgenes amplios y seguimiento estricto logrando una resolución dramáticamente satisfactoria. De no localizarse el tumor el tratamiento es en base a aportes de fosfato y calcitriol.

Cabe destacar que el FGF23 ha contribuido a un creciente diagnóstico de esta enfermedad, tanto así que de los aproximados 300 casos de TIO reportados en la literatura dos tercios han sido descritos en los últimos 10 años.

Pese a ser una enfermedad infrecuente y rara, tanto los clínicos como los patólogos deben tener presente esta entidad, logrando así un diagnóstico y tratamiento precoz con beneficios sustanciales para el paciente.

\section{BIBLIOGRAFÍA}

1. Bisceglia M, Spagnolo D, Galliani C y cols. Tumoral, quasitumoral and pseudotumoral lesions of the superficial and somatic soft tissue: new entities and new variants of old entityes recorded during the last 25 years. Part V: Excerpta III. Pathologica 2004; 96: 481-95.

2. Thompson LDR, Miettinen M, Wenig BM. Sinonasaltype hemangiopericytoma. A clinicopathologic and immunophenotypic analysis of 104 cases showing perivascular myoid differentiation. $\mathrm{Am}$ J Surg Pathol 2003; 27: 737-49.

3. Folpe AL, Fanburg-Smith JC, Billings SD y cols. Most osteomalacia-associated mesenchymal tumors are a single histopathological entity. $A m$ J Surg Pathol 2004; 28: 1-30.

4. Weiss D, Bar RS, Weidner N, Wener M, Lee F. Oncogenic osteomalacia: strange tumors in strange places. Postgrad Med J 1985; 61: 349-55.

5. Sundaram M, McCarthy EF. Oncogenic osteomalacia. Skeletal Radiol2000; 29: 117-24.

6. De Beur SM, Finnegan RB, Vassiliadis y cols. Tumors associated with oncogenic osteomalacia express genes important in bone and mineral metabolism. $J$ Bone Miner Res 2002; 17: 1102-10.

7. Shimada T, Mizutani S, Muto T y cols. Cloning and characterization of FGF23 as a causative factor of tumor-induced osteomalacia. Proc Natl Acad Sci USA 2001; 98: 6500-5.

8. RAmON I, KLEYNen P, BOdY JJ Y COLS. Fibroblast growth factor 23 and its role in phosphate homeostasis [J]. Eur J Endocrinol 2009; 162 (1): 1-10. 
9. McCance RA. Osteomalacia with Looser's nodes (Milkman's syndrome) due to raised resistance to vitamin $D$ acquired about the age of 15years. Quarterly J Med 1947; 16: 33-47.

10. Ryan EA, Reiss E. Oncogenus osteomalacia. Review of the world literature of 42 cases and report of two new cases. Am J Med 1984; 77: 501-12.

11. Guillem Viscasillas, Javier Maiz. Osteomalacia oncogénica por tumor mesenquimal fosfatúrico en fosa infratemporal. Acta Otorrinolaringol Esp 2010; 61(5): 392-4.

12. Saito H, Kusano K, Kinosaki M, Ito H, Hirata M, SEgaWA H Y COLS. Human fibroblast growth factor23 mutants suppress NA dependent phosphate co-transport activity and 1,25-dihydroxyvitamin D3 production. J Biol Chem 2003; 278: 2206-11.

13. Giuseppe Guglielmi, Michele Bisceglia, Alfredo Scillitani, Andrew L. Folpe. Oncogenic osteomalacia due to phosphaturic mesenchymal tumor of the craniofacial sinuses. Clin Cases Miner Bone Metab 2011; 8(2): 45-9.

14. Folpe AL, Fanburg-Smith JC, Billings SD, Bisceglia M, Bertoni F, Cho JY y cols. Most osteomalaciaassociated mesenchymal tumors are a single histopathologic entity: an analysis of 32 cases and a comprehensive review of the literature. $A m$ J Surg Pathol 2004; 28: 1-30.

15. Takashi Shimada, Makoto Kakitani, YuJ Yamazaki Y coLs. Targeted ablation of Fgf23 demonstrates an essential physiological role of FGF23 in phosphate and vitamin D metabolism. Clin Invest 2004; 113(4): 561-8.

16. Linsey M, Smith W, Yamauchi H, Bernstein L. Nasopharyngeal angiofibroma presenting as adult osteomalacia: case report and review of the literature. Laryngoscope 1983; 102: 869-70.

17. Weidner N, Santa Cruz D. Phosphaturic mesenchymal tumors: a polymorphous group causing osteomalacia or rickets. Cancer 1987; 59: 1442-54.

18. Papottı M, Foschini MP, Isaia G, Rizzı G, Betts CM, EusebI V. Hypophosphatemic oncogenic osteomalacia: report of three new cases. Tumori 1988; 74: 599-607.
19. Wilkins Ge, Granleese S, Hegele RG, Holden J, ANDERSON DW, Bondy GP. Oncogenic osteomalacia: evidence for a humoral phosphaturic factor. J Clin Endocrinol Metab 1995; 80: 1628-34.

20. González-Compta X, Mañós-Pujol M, FogliaFeRnÁndez M Y cols. Oncogenic osteomalacia: case report and review of head and neck associated tumours. J Laryngol Otol 1998; 112: 389-92.

21. OHAShI K, OHNISHI T, IshiKaWa T, TanI H, Uesugi K, TAKAGI M. Oncogenic osteomalacia presenting as bilateral stress fracture of the tibia. Skeletal Radiol 1999; 28: 46-8.

22. Clunie GP, Fox PE, Stamp TC. Four cases of acquired hypophosphatemic ('oncogenic') osteomalacia: problems of diagnosis, treatment and long-term management. Rheumatology (Oxford) 2000; 39: 1415-21.

23. Sandhu FA, Martuza RL. Craniofacial hemangiopericytoma associated with oncogenic osteomalacia: case report. J Neurooncol 2000; 46: 241-7.

24. John MR, Wickert $\mathrm{H}$, ZaAr $\mathrm{K}$ y cols. A case of neuroendocrine oncogenic osteomalacia associated with a PHEX and fibroblast growth factor-23 expressing sinusoidal malignant schwannoma. Bone 2001; 29: 393-402.

25. William H Chong, Alfredo A Molinolo, Clara C Chen and Michael T Collins. Review: Tumor-induced osteomalacia. Endocr Relat Cancer 2001; 18(3): R53-R77.

26. Florencia Halperin, Ronald J Anderson and Jean E Mulder. Tumor-induced osteomalacia: the importance of measuring serum phosphorus levels. Nature Clinical Practice Endocrinology \& Metabolism 2007; 3: 721-5.

27. Yoshioka K, NAGata R, UEDA M, y cols. Phosphaturic mesenchymal tumor with symptoms related to osteomalacia that appeared oneyear after tumorectomy [J]. Intern Med 2006; 45(20): 1157-60.

28. Hannan FM, Athanasou NA, Teh J, y cols. Oncogenic hypophosphataemic osteomalacia: biomarker roles of fibroblast growth factor 23 , 1,25_di hydroxyvitamin D3 and lymphatic vessel endothelial hyaluronan receptor 1 [J]. Eur J Endocrinol 2008; 158(2): 265-71.

Dirección: José Tomás Andrade D.

Asturias 171, Las Condes

E mail: tomasandrade@gmail.com 